\title{
Localization of License Plates from Surveillance Camera Images: A Color Feature Based ANN Approach
}

\author{
Satadal Saha \\ Sr. Lecturer \\ CSE Department \\ MCKV Institute of Engg. \\ Liluah
}

\author{
Subhadip Basu \\ Sr. Lecturer \\ CSE Department \\ Jadavpur University \\ Kolkata
}

\author{
Mita Nasipuri \\ Professor \\ CSE Department \\ Jadavpur University \\ Kolkata
}

\author{
Dipak Kr. Basu \\ AICTE Emeritus Fellow \\ CSE Department \\ Jadavpur University \\ Kolkata
}

\begin{abstract}
Traffic monitoring system has now become an essential administrative part in most of the developed and developing countries. In general, such systems monitor/identify the vehicles exceeding speed limits, or monitor the vehicles crossing the stop line at red traffic signal. It may also be used for registering the vehicles getting entry in a shopping mall or in a railway station or in an airport. The key modules of these monitoring systems are: (i) localization of license plates within the image and (ii) recognizing the license number using an OCR system. The present work addresses the first module of the system. The color information of the license plate is used as the knowledge base for training an artificial neural network system using back propagation algorithm. The trained network is then used to find the potential license plate region within a new traffic image. The scheme is applied in a real life outdoor environment at some road crossings in an Indian city. The result is found to be quite satisfactory giving an accuracy of around $80 \%$.
\end{abstract}

\section{Categories and Subject Descriptors}

H.2.0 [IMAGE]: Color Image Processing - RGB to HSI conversion, Sobel edge operator, Hough transform.

\section{General Terms}

Algorithms, Performance, Design, Experimentation

\section{Keywords}

RGB-HSI, Back propagation algorithm, connected component labeling, Sobel edge detector, Hough transform

\section{INTRODUCTION}

Traffic monitoring system has long been an active area of research because of its wide application in managing signal violations at crowded road crossings or automatic registration of vehicle in public places. License plate localization plays an important role as the first module of such system. The localization task becomes more challenging in an unconstrained outdoor environment, especially when there is a wide variation of the size, shape and color of the license plate. The non- standardization of the character set of the license plate further complicates the recognition procedure by manifolds.

Various techniques have so far been developed for the purpose of efficient detection of license plate regions from offline vehicular images. In one of the earlier works, rank filter is used by Martinsky [1] for localization of license plate regions. However, the technique gives poor results for skewed license plates. An analysis of Swedish license plates is reported in [2] by Bergenudd using vertical edge detection, followed by binarization. This technique does not give desired results for non-uniformly illuminated license plates. In [3], license plate characters are localized and the width, height, area and the aspect ratio are used as parameters in a genetic algorithm based optimisation technique for selecting the license plate region. The approach is highly dependent on the proper localization of the plate characters and fails if the image contains almost no edge near the license plate boundary. License plate localization and recognition techniques are developed in [4] using attributes of the plates and neural network. The technique then analyses syntax of it to get the correct license plate. The method gives efficient localization but poor recognition results. A work on Iranian license plate localization is done in [5]. It uses Sobel's operator to get the vertical edge image, Otsu's method to binarize the image and also some morphological operations for localizing the license plate. It is a robust method and is rotation/ scale invariant and also applicable to different lighting conditions. In [6], mean shift algorithm is used for license plate localization. The rectangularity attribute, aspect ratio and edge density are used as feature in Mahalanobis distance based classifier for detecting the correct license plate. The method is robust as long as the color of the license plate differs from the color of the body of the vehicle, at which it adheres to. Spanish license plate localization and recognition is done in [7] using horizontal Sobel's operator in the low saturation areas of the image. The aspect ratio and the distance of the potential license plate regions from the center of the image are subsequently considered as the features to identify the correct license plate. The method is independent of the size, position and orientation of the license plate and also independent of the lighting conditions. But the technique is applicable for license plates with white background and black characters only. Sliding concentric windows is used in [8] for faster detection of region of interest and artificial neural network (ANN) is then used 
for the recognition purpose. In another approach almost horizontal license plates are localized and recognized in [9] using ANN.

Not much work has been done on localization of the license plates for Indian vehicles. A reference on ALPR of Indian vehicles is found in [10]. However, the technical details and the performance of such techniques could not be evaluated on real life datasets. In one of our earlier works, a color based segmentation scheme is reported in [11] for Indian commercial vehicles. In another work, vertical edge density of the characters is used in [12] for localization of license plate. However, both of our earlier techniques had limited generalization attributes. A technique for identifying the images in which the vehicles have actually violated the traffic red signal has already been reported in [13]. In the following two sections we have discussed the collection of dataset and the basic methodologies employed for the present work. In the subsequent sections the experimental results and the conclusions are discussed.

\section{COLLECTION OF DATASET}

The dataset for the current experiment is collected as a part of demonstration project of a government traffic monitoring system at a busy crossing in a city. Two surveillance cameras were installed at two sides of the road at about ten meters height. All the cameras were focused at the stop line of the road so that it can capture the frontal view of the vehicle. The cameras were synchronized with the traffic signal system such that it starts capturing the images when the signal is RED. The CCD cameras captured images day and night at different unconstrained environment, at different lighting conditions, wind turbulence etc. The complete dataset contains more than 5000 images of size 704 $\times 576$ pixels. Out of these images only 500 images are found, which contain clear view of the license plate region and these images are considered for this experiment.

\section{PRESENT WORK}

In the present work, we have developed an artificial neural network based technique which is trained with color features of the characters of the license plate. The trained network is then used to localize the license plate in the video snapshots captured from the surveillance cameras. In the subsequent sections the key modules involved in the present work has been discussed.

\subsection{Training the neural network}

\subsubsection{Designing the neural network}

In the present work multilayer perceptron is used, with one input layer, one output layer and one hidden layer. As we have used thirty features of each of the characters of the license plate, the number of nodes in the input layer is 30 . The output layer contains two nodes: one for license plate region and the other for the no-plate region. So it is a two-class problem. The number of nodes in the hidden layer is varied within a range and the training data for which the performance is best is stored in a file.

\subsubsection{Generating the training dataset}

From the large number of images we have in the dataset, the characters are cut from the license plate and are stored as separate file. In this way a dataset of license plate characters only is created. All these images are designated as class 0 data. Similarly, character-size portions are cut from the no-plate region of the images and are stored as files. In this way, a dataset of no-plate region is generated. All these images are designated as class 1 data. For both class 0 and class 1 data the image is divided in quin tree structure generating five cells. For each of the cells color based 30 features are computed. Each pixel has red (R), green $(\mathrm{G})$ and blue (B) components of its color value. These values are used to get the corresponding hue $(\mathrm{H})$, saturation $(\mathrm{S})$ and intensity (I) components using the following formula.

$$
\begin{aligned}
& H=\begin{array}{cc}
\theta & \text { if } B \leq G \\
360-\theta & \text { if } B \leq G
\end{array} \\
& \theta=\cos ^{-1}\left[\begin{array}{c}
\frac{1}{2}[(R-G)+(R-B)] \\
{\left[(R-G)^{2}+(R-B)(G-B)^{6}\right.}
\end{array}\right] \\
& S=1-\frac{3}{(R+G+B)}[\min (R, G, B)] \\
& I=\frac{1}{3}(R+G+B)
\end{aligned}
$$

For each cell following features are computed:

(i) Mean of R, G, B, H, S, I: These are six features carrying the mean of each of the R, G, B, H, S, I components

(ii) UpperMean of R, G, B, H, S, I: These are six features carrying the mean of each of the R, G, B, H, S, I components considering the cases where the particular color component is greater than the corresponding mean value.

(iii) LowerMean of $\mathrm{R}, \mathrm{G}, \mathrm{B}, \mathrm{H}, \mathrm{S}, \mathrm{I}$ : These are six features carrying the mean of each of the R, G, B, H, S, I components considering the cases where the particular color component is lower than the corresponding mean value.

(iv) Max of R, G, B, H, S, I: These are six features carrying the maximum of each of the R, G, B, H, S, I components

(v) Min of R, G, B, H, S, I: These are six features carrying the minimum of each of the R, G, B, H, S, I components

All the above mentioned R, G, B, H, S, I values are divided by their corresponding maximum values to get the fractional components (r, g, b, h, s, i). These fractional components are used as feature values. The above features are computed for each cell corresponding to each image thus generating 5 data for the particular image. In this way, all the images are used to extract the features and are stored in a file. For both classes, the dataset generated are used for training the network and for also for testing it. So the dataset is divided into two parts: $80 \%$ of the data for both the classes are stored in the training file and the remaining $20 \%$ data for both the classes are stored in the test file. The test file is used for getting the efficiency of the trained neural network when applied on the test dataset. 


\subsubsection{Training ANN using Back Propagation Algorithm}

The hidden and output layer perceptron weights are initialized with random numbers between 0 and 1 . In the process of training through back propagation algorithm, the network reads the feature values from the training dataset, gets the output for the feature values, computes the error by calculating the difference from the desired output and back propagates the error to adjust the weight factors. These actions are done repeatedly for a predefined number of iterations and for a particular number of hidden layer neurons. Intermediately, the maximum accuracy thus obtained for a specific iteration is stored in the network file. In the same way, the network is trained for a specific set of hidden layer neuron values and for each such value the trained network is used to find the output efficiency for the test dataset. The network data giving maximum accuracy is used for localization of license plate.

\subsection{Localization of license plate}

The view angle of the image plays an important role in the localization process. The frontal face of the camera normally does not remain parallel to the frontal face of the vehicle. As a result the license plates within the image remain skewed. The skewness is corrected by rotating the image by a certain angle either clockwise or anticlockwise. The skew angles for all the images captured by a particular camera always remain fixed, as it is dependent only on the fixation of the camera itself. The rotated image containing the license plate is subdivided into ' $n$ ' number of cells, each having a dimension approximately close to the character size. Some of the cells will obviously contain the part of license plate and the rest and certainly most of the cells will contain no-plate information. For each cell, the 30 features discussed in section 3.1.2 are computed and are stored in a test data file. The already trained neural network then reads the network file and sets different parameters of it according to the values read from the file. It then reads the test data file and for each feature data corresponding to each cell it generates output class values. The highest output class value is the indication of the class as predicted by the neural network system.

The cells that are predicted as class 0 , i. e. license plate region, by the network are marked as ' 1 '. The marked cells are then passed to "noise cell remove()" module, the primary task of which is to remove isolated marked cells placed here and there in the image. These cells are actually identified as noise cells and can not be part of license plate. The outcome of the module is some selected cells, which are then fed to "ccl()" module which runs connected component labeling algorithm to generate segments with potential license plate region. At this stage a filtration technique is applied on the segments depending on the aspect ratio and the area of the segments to reject very small sized segments. It is seen that the segments still may not contain the license plate, or even there may not be any character like appearance in the segments. To locate the actual segments finally Hough transform is applied over the segments. First horizontal Hough transform is applied on the edge image of the individual segments and then on the Hough image, the vertical Hough transform is applied.

\section{EXPERIMENTAL RESULTS}

The method described in section 3 is used to find the license plate region within a dataset of 219 unique images. Fig. 1. shows the result of accurate localization of license plate. Fig. 1 (a-c) show accurate localization of license plates from the images capture at night. The system is also capable of detecting any number of license plates within an image. Fig. 1 (h) shows the result of detecting two license plates within an image. Fig. 2. shows images where localization region is more than the actual size of the license plate. Fig. 3. shows images where false localization has been resulted along with true localization of license plate. The system has the inherent tendency of locating any text within the image, head light of the vehicle or the grid like protector of the head light. Fig. 4. shows the results where the false localization has only been generated. This may be due to the very poor quality of the license plate characters or due to the reflection of sun light from the license plate. 


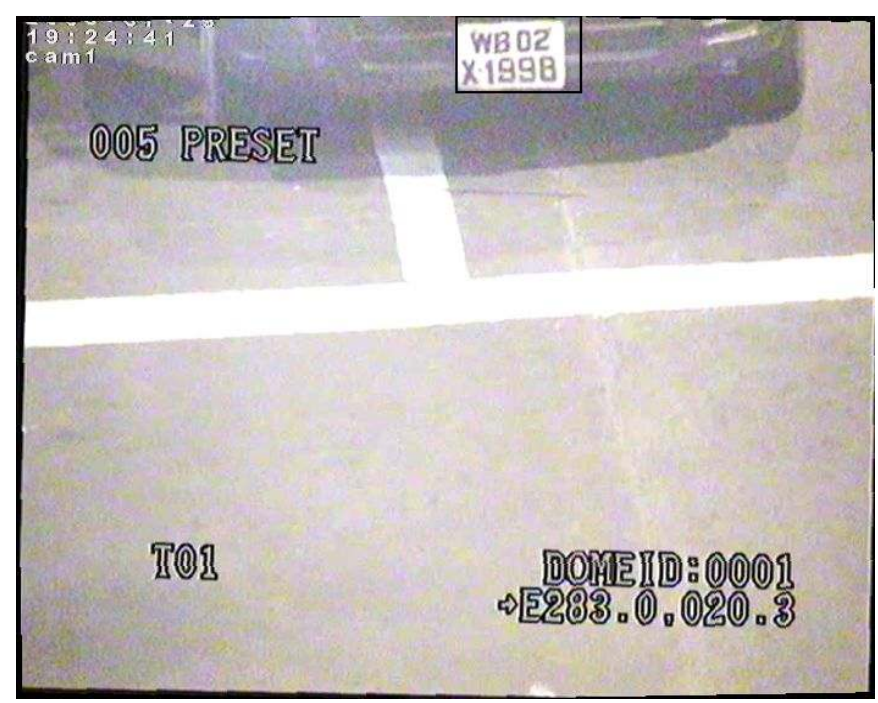

(a)
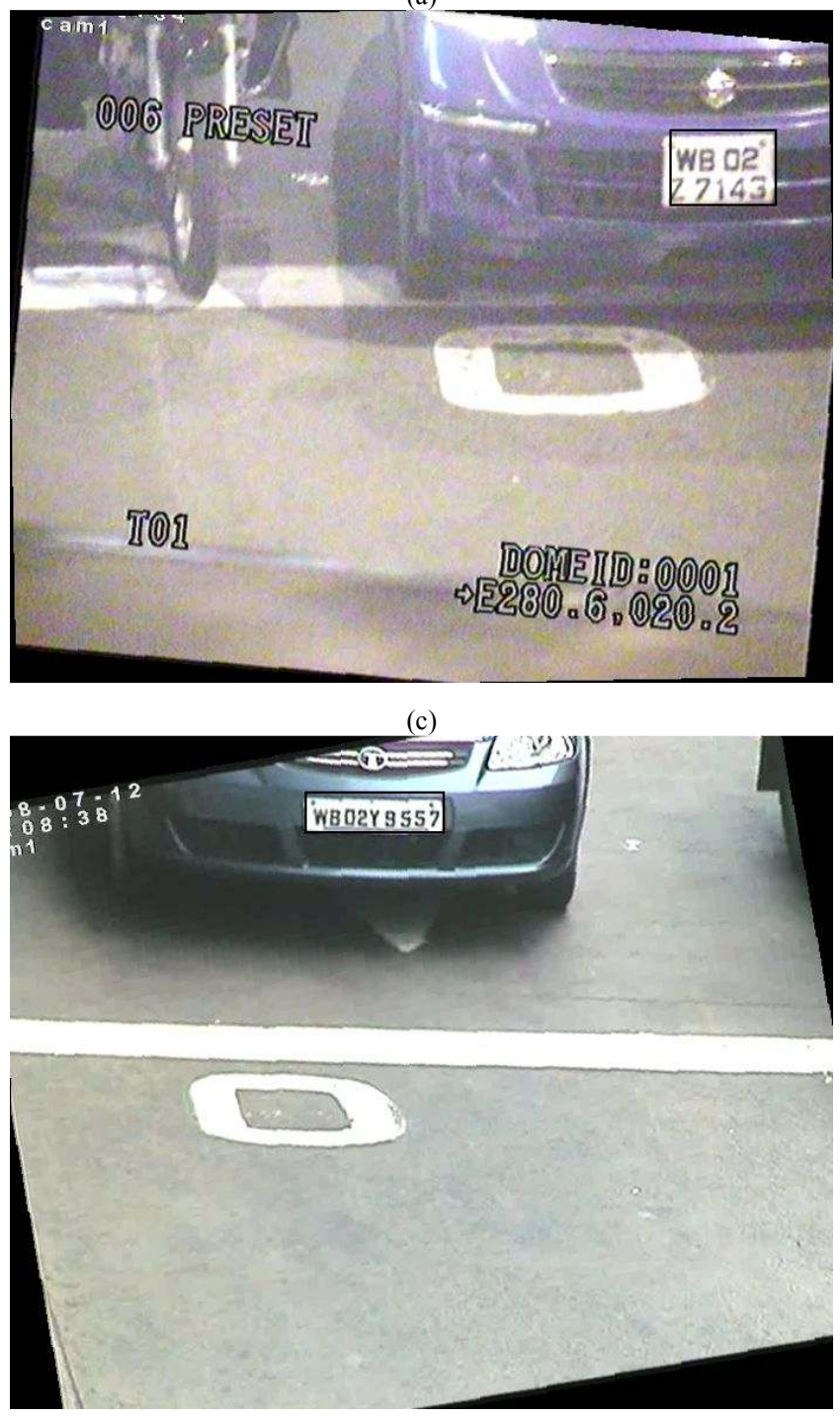

(e)

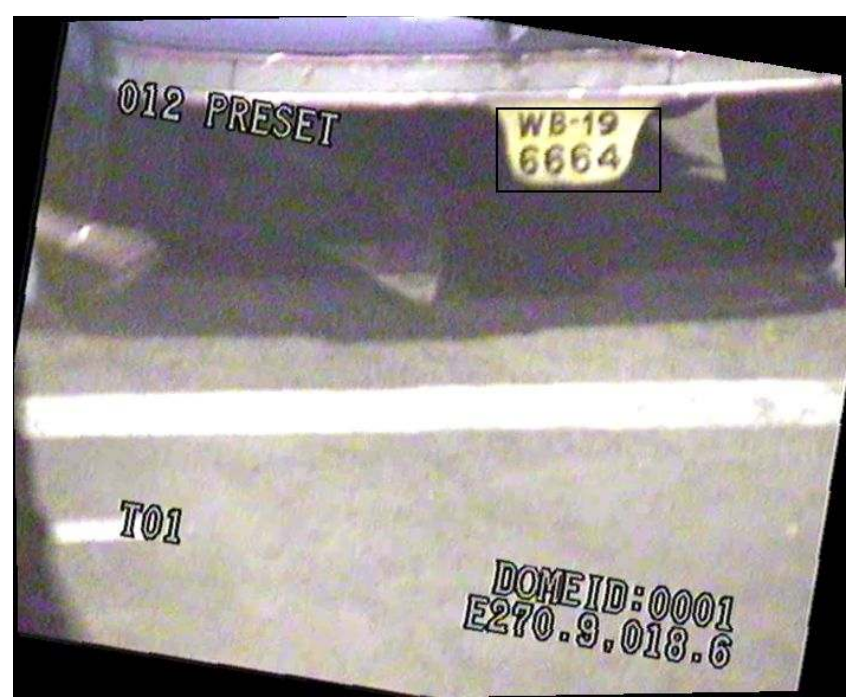

(b)
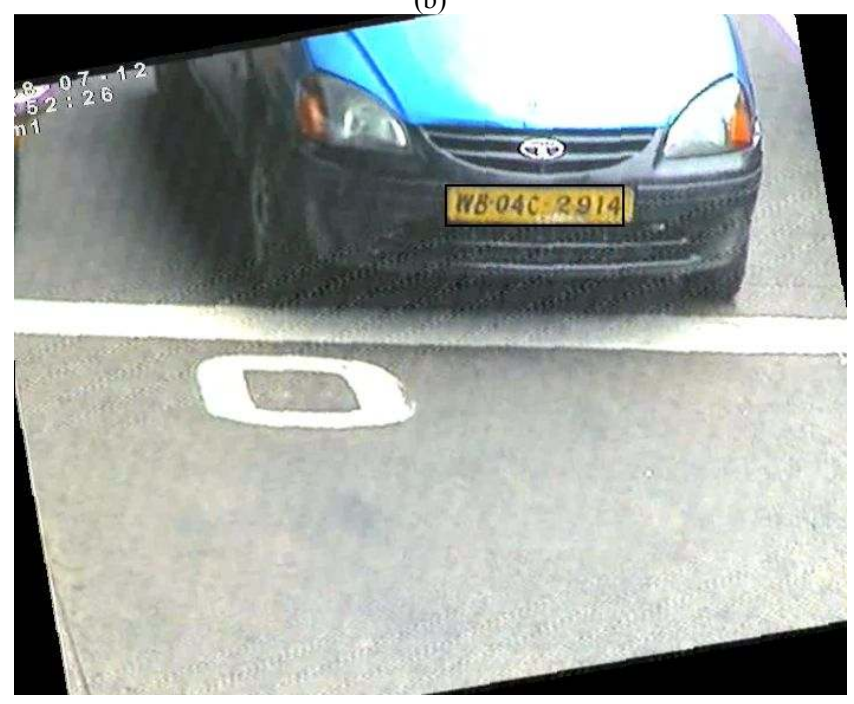

(d)
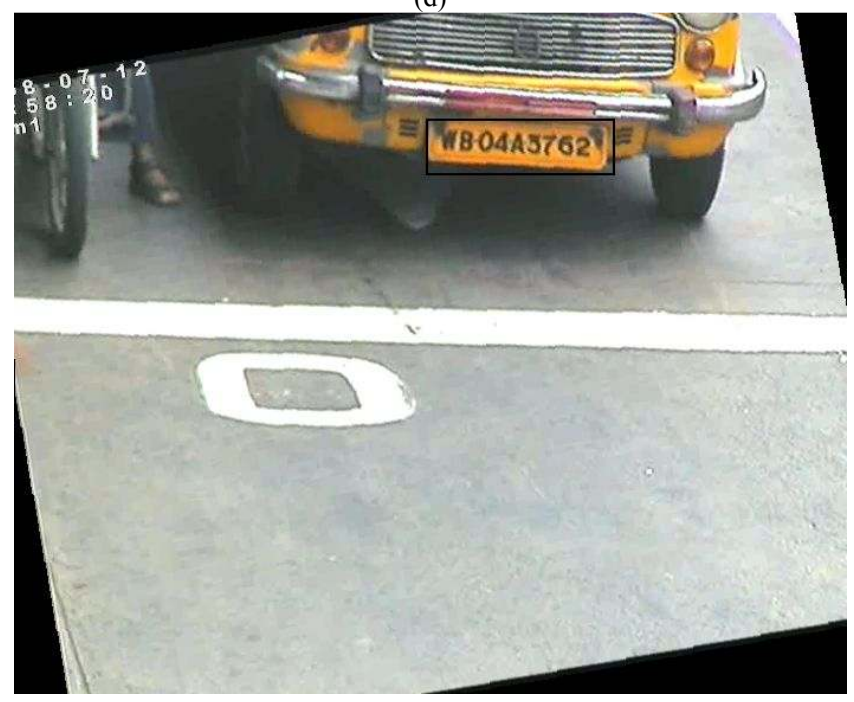

(f) 


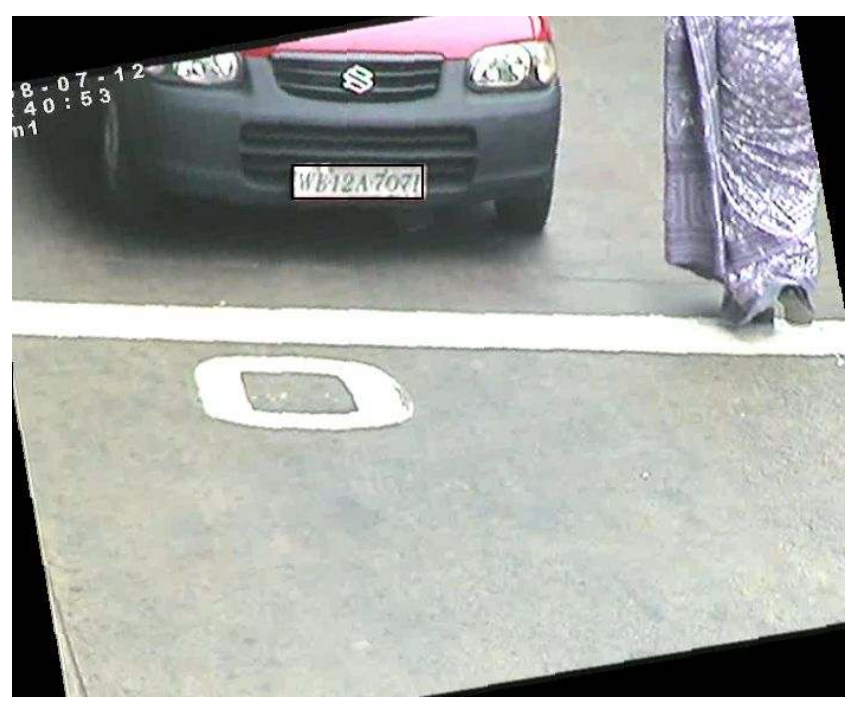

(g)

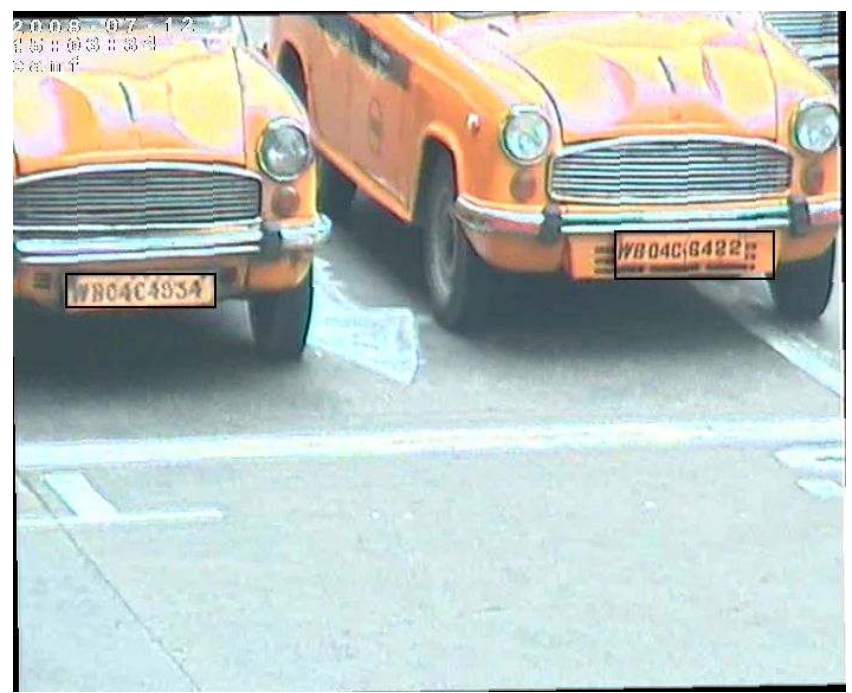

(h)

Figure 1. Accurate Localization of License Plate

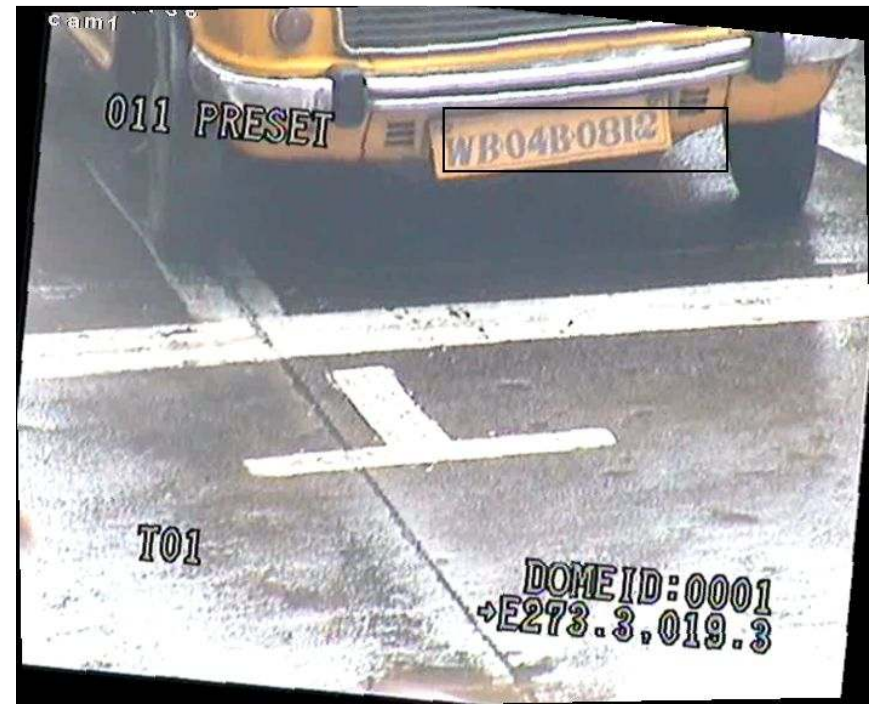

(a)
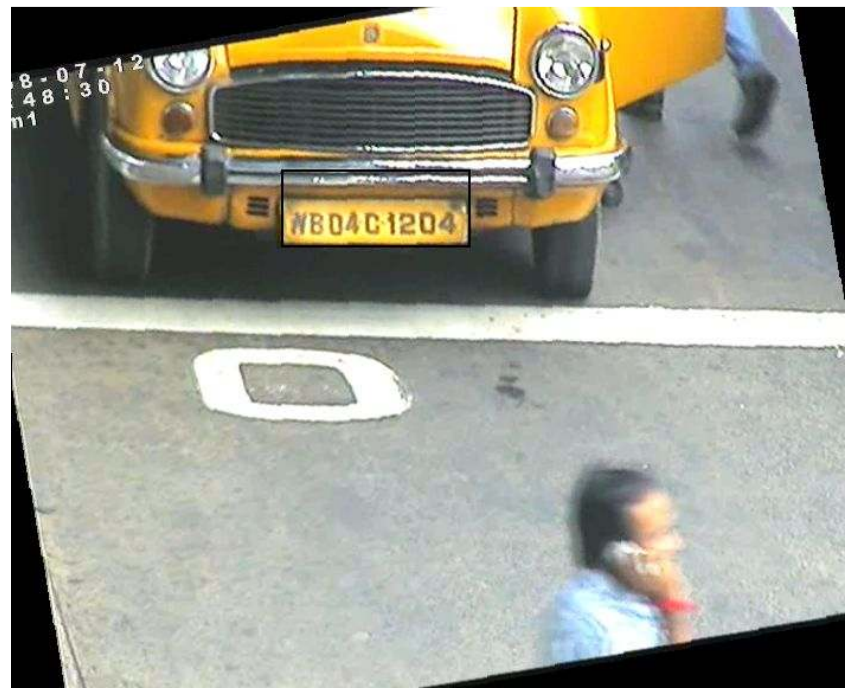

(b)

Figure 2. Extended localization region 


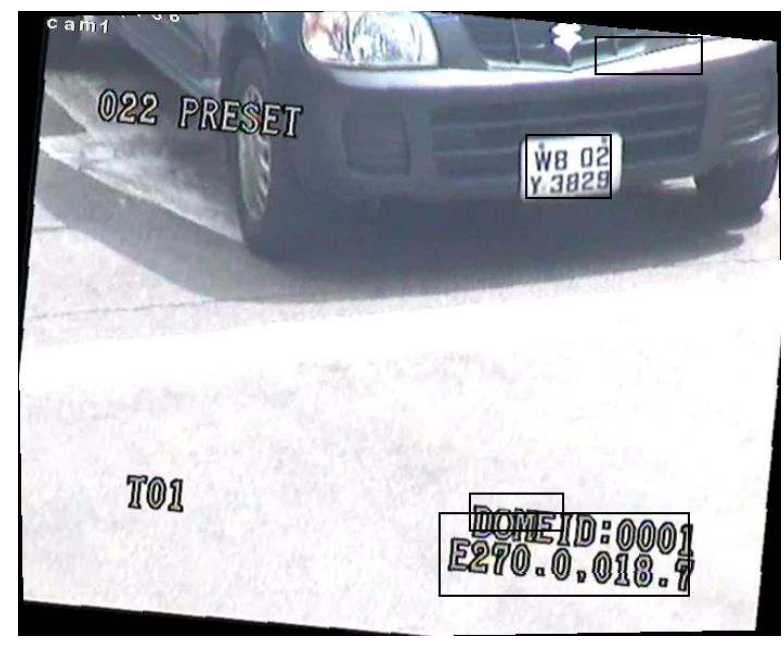

(a)

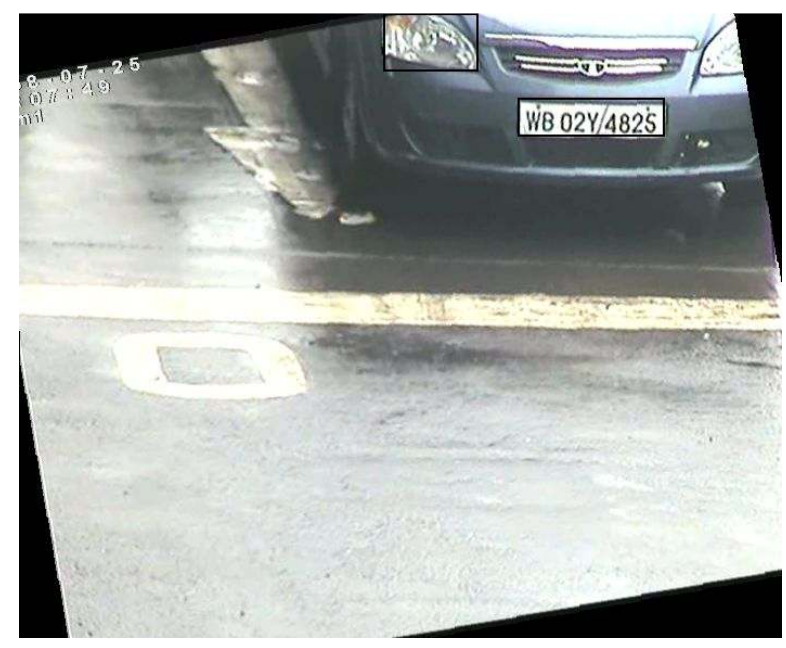

(c)

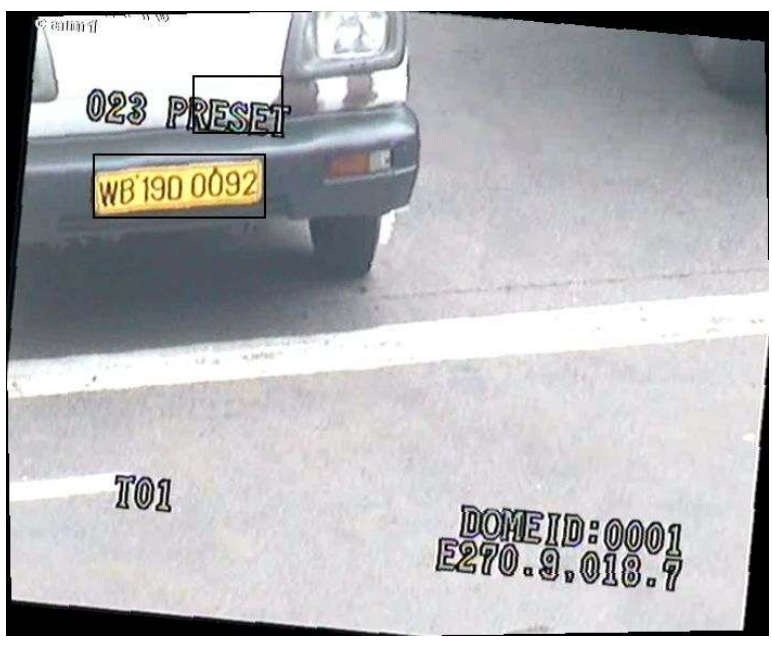

(b)

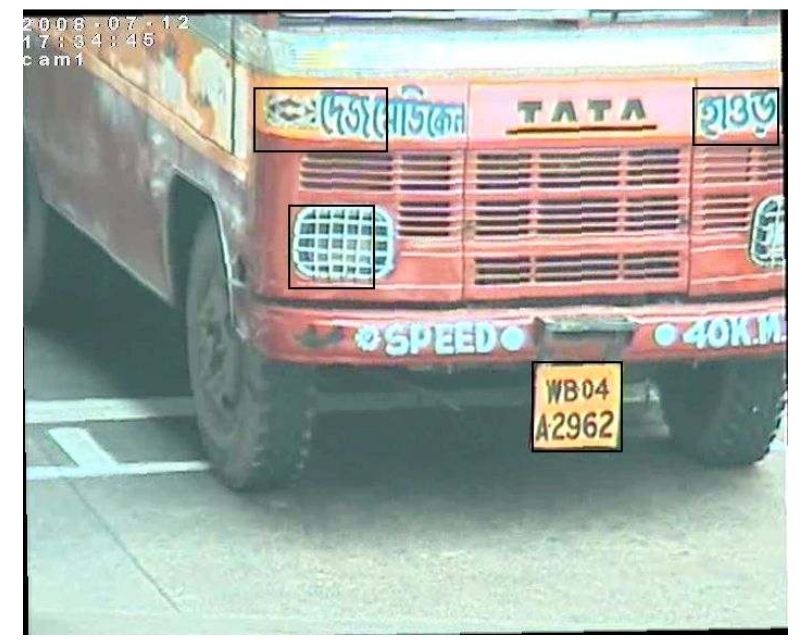

(d)

Figure 3. False along with true localization of license plate

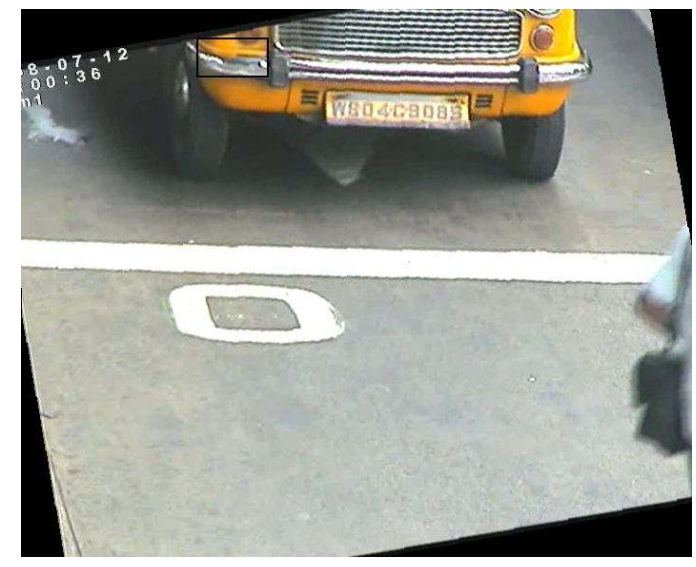

(a)
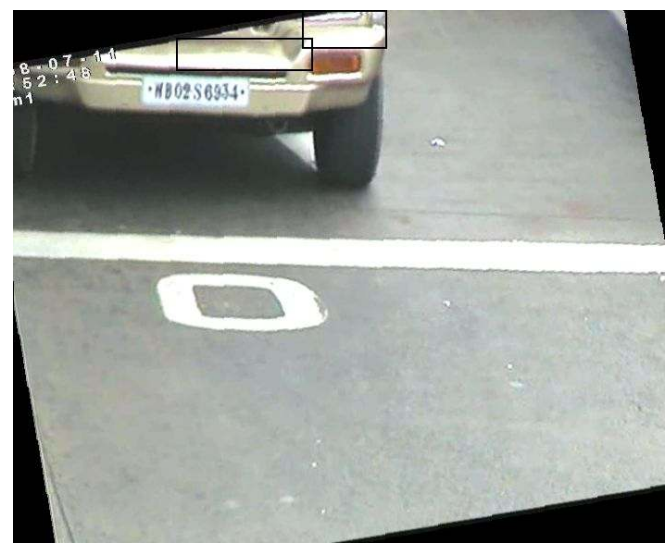

(b)

Figure 4. False localization of license plate 


\section{CONCLUSION}

It is evident from the above discussion that the current technique efficiently identifies potential license plate regions from the surveillance-camera captured vehicle images. The technique successfully employs a RGB-HSI colour feature based neural network approach for the said purpose. The performance is evaluated on real datasets, collected from one of our implemented/working software modules, developed for a city traffic monitoring authority of a Metropolitan city in India. The performance of the technique is found to be satisfactory and implementable on real systems with actual deliverables. The work may further be extended for designing a effective license plate segmentation system for actual recognition of characters.

\section{ACKNOWLEDGMENTS}

Authors are thankful to the CMATER and the SRUVM project, C.S.E. Department, Jadavpur University, for providing necessary infrastructural facilities during the progress of the work. One of the authors, Mr. S. Saha, is thankful to the authorities of MCKV Institute of Engineering for kindly permitting him to carry on the research work.

\section{REFERENCES}

[1] O. Martinsky, "Algorithmic and Mathematical Principles of Automatic Number Plate Recognition System", B. Sc. Thesis, BRNO University of Technology, 2007.

[2] Erik Bergenudd, "Low-Cost Real-Time License Plate Recognision for a Vehicle PC", Master's Degree Project, KTH Electrical Engineering, Sweden, December 2006.

[3] J. R. Parker and P. Federl, "An Approach to License Plate Recognition”, Computer Science Technical Report (1996591-1. I), 1996.

[4] H. Kawasnicka and B. Wawrzyniak, "License Plate Localization and Recognition in Camera Pictures", AIMETH 2002, Poland, November 2002.

[5] H. Mahini, S. Kasaei, F. Dorri and F. Dorri, “An Efficient Features-Based License Plate Localization Method", Proceedings of $18^{\text {th }}$ International Conference on Pattern Recognition, 2006.
[6] W. Jia, H. Zhang, X. He and M. Piccardi, "Mean Shift for Accurate License Plate Localization", Proceedings of $8^{\text {th }}$ International IEEE Conference on Intelligent Transportation Systems, Vienna, Austria, Sept. 2005.

[7] Cesar Garcia-Osorio, Jose-Francsico Diez-Pastor, J. J. Rodriguez, J. Maudes, "License Plate Number Recognition New Heuristics and a comparative study of classifier", cibrg.org/documents/Garcia08ICINCO.pdf.

[8] C. N. Anagnostopoulos, I. Anagnostopoulos, V. Loumos and E. Kayafas, "A license plate recognition algorithm for Intelligent Transport applications", www.aegean.gr/culturaltec/canagnostopoulos/cv/T-ITS-0508-0095.pdf.

[9] S. Draghichi, "A neural network based artificial vision system for license plate recognition", International Journal for Neural Systems, Vol. 8, 1997, pp. 113-126.

[10] V. S. L. Nathan, Ramkumar. J, Kamakshi. P. S, "New approaches for license plate recognition system", ICISIP 2004, p.p. 149-152.

[11] Satadal Saha, Subhadip Basu, Mita Nasipuri and Dipak Kumar Basu, "An Offline Technique for Localization of License Plates for Indian Commercial Vehicles", Proceedings of IEEE National Conference on Computing and Communication Systems (COCOSYS-09), UIT, Burdwan, January 02-04, 2009, pp. 206-211.

[12] Satadal Saha, Subhadip Basu, Mita Nasipuri and Dipak Kumar Basu, "License Plate localization from vehicle images: An edge based multi-stage approach", In press International Journal on Recent Trends in Engineering (Computer Science), Vol. 1, No. 1, 2009, pp. 284-288.

[13] Satadal Saha, Subhadip Basu, Mita Nasipuri and Dipak Kumar Basu, "Development of an Automated Red Light Violation Detection System (RLVDS) for Indian vehicles", Proceedings of IEEE National Conference on Computing and Communication Systems (COCOSYS-09), UIT, Burdwan, January 02-04, 2009, pp. 59-64.

[14] R. C. Gonzalez and R. E. Woods, Digital Image Processing, Second Edition, Pearson Education Asia, 2002. 